\title{
Deformation of impulse response estimates by time window in cross spectral technique
}

\author{
Ken'iti Kido, ${ }^{*}$ Hideo Suzuki, ${ }^{*}$ Takahiko Ono, ${ }^{* *}$ and Manabu Fukushima ${ }^{* * *}$ \\ * ONTEK R\&D Co. Ltd., \\ 1-16-1, Hakusan, Midori-ku, Yokohama, 226-8507 Japan \\ ** ONO SOKKI Co. Ltd., \\ 1-16-1, Hakusan, Midori-ku, Yokohama, 226-8507 Japan \\ *** Chiba Institute of Technology, \\ 2-17-1, Tsudanuma, Narashino, 275-0016 Japan
}

(Received 17 November 1997)

\begin{abstract}
The estimation of transfer function and impulse response of a system by the cross spectral technique is very popular and most of the FFT analyzer is equipped with that function. In the cross spectral technique, the impulse response changes its form by the use of time window. But the deformation of the impulse response in the process of estimation is not yet well known. This paper first theoretically describes why and how the impulse response changes its form. According to the theoretical derivation, the deformation of the impulse response is illustrated for several commonly used time windows and the results are verified by the computer simulation.
\end{abstract}

Keywords: Impulse response, Transfer function, Time window, Convolution, Cross spectrum

PACS number: 43. 60. - c, 43. 60. Gk, 43. 58. Kr, 43. 58. Ta

\section{INTRODUCTION}

The exact estimation of the transfer function and the impulse response is necessary in various applications. Several estimation methods were proposed except for the use of the cross spectral technique. The exact impulse response can be estimated by the time-stretched pulse (TSP) method ${ }^{1,2)}$ or by the many time averaging method. ${ }^{3)}$ But, the TSP method requires the well prepared source signal and the averaging method requires the random sequence whose averaged value is zero or exact impulse to drive the transfer system.

On the contrary, the condition required for the source signal in the cross spectral technique is only not to have zero point in the averaged power spectrum. The property of circular convolution in the cross spectrum can be effectively used for the estimation of impulse response by the cross spectral tech- nique. That is, the exact impulse response is obtained using the periodic signal which is composed of the sinusoidal wave of the same period as the window length and all the harmonics of the wave. But, such the source signal can not be always used. Therefore, the cross spectral technique using random sequence as the source signal has widely been used for the estimation of the transfer function and the impulse response of a system.

The time window is necessarily used for the computation of the discrete Fourier transform (DFT) in the cross spectral technique as the DFT is the Fourier transform of a finite number of data. The fast Fourier transform (FFT) algorithm is used for the computation of the DFT. An identical time window is usually used to cut out the sampled data in the input and the output sequences.

The time window plays an important role in the cross spectral technique. But, most of the investiga- 
tions on the time windows so far carried out concerned mainly with the power spectrum estimation. $\left.{ }^{4}\right)$ The group of one of the authors investigated on the effect of time window on the estimation of coherence function and impulse response by cross spectral technique in the view point of estimation error $^{5-7)}$ but how the impulse response changes was not made clear.

This paper explains the effect of the time window on the envelope of the impulse response estimated by the cross spectral technique using a random sequence as the source signal. The result is verified through the computer simulation.

\section{PROBLEMS IN THE CROSS SPECTRAL TECHNIQUE}

Using $n$ as the discrete time, the response $y(n)$ to an input sequence $x(n)$ of a system having the impulse response $h(n)$ is the linear convolution of $x(n)$ and $h(n)$ which is usually expressed by the following equation.

$$
y(n)=x(n) * h(n)+n(n)
$$

where $n(n)$ is the external noise which is neglected in this paper as it has no relation to the present discussion.

Then we start from the next equation.

$$
y(n)=x(n) * h(n)
$$

There is the possibility of confusing the linear convolution and the circular convolution in the expression of Eq. (2), that is, the equation having such the form is sometimes seemed to be the expression of the circular convolution though the convolution of $x(n)$ and $h(n)$ is practically the linear convolution as mentioned above.

The Fourier transform of Eq. (2) can superficially be written as follows:

$$
Y(k)=X(k) H(k)
$$

where, the functions of $k$ expressed by the capital letters denote the Fourier transforms of the corresponding time domain functions expressed by the lower-case letters and $k$ the discrete frequency.

Multiplying the complex conjugate of the DFT of $x(n)$ to the both side of Eq. (3), the following relation is made.

$$
X(k)^{*} Y(k)=X(k)^{*} X(k) H(k)
$$

By the many time averaging, the cross spectrum of the input and the output sequences is computed as follows.

$$
\begin{aligned}
S_{X Y}(k) & =\overline{X(k)^{*} Y(k)}=\overline{X(k)^{*} X(k)} H(k) \\
& =S_{X X}(k) H(k)
\end{aligned}
$$

where, $\quad S_{X Y}(k)$ is the cross spectrum of $x(n)$ and $y(n)$,

$S_{X X}(k)$ is the power spectrum of $x(n)$ and indicates many time averaging.

From Eq. (5), the transfer function $H(k)$ of the system is expressed as follows.

$$
H(k)=\frac{S_{X Y}(k)}{S_{X X}(k)}
$$

The above deduction is superficially correct, but it can not be correct as long as Eq. (3) is the DFT of a finite number of data which expresses the circular convolution of the finite numbers of $x(n)$ and $h(n)$.

Moreover, the input and the output sequence is usually multiplied by a time window function $w(n)$ in the cross spectral technique. When a time window is used, $X(k)$ should be changed to $X(k) *$ $W(k)$ the convolution of $X(k)$ and the DFT of $w(n)$ because the Fourier transform of the product of two time domain functions is the convolution of the spectrum of each time domain function. $Y(k)$ should be also changed to $Y(k) * W(k)$ by the same reason. Such the problem has not been accounted in the conventional deconvolution method by use of the cross spectral technique.

Here, we deal with the output sequence as the sum of the responses to each sample in the input sequence based on the assumption that the system is linear.

We denote the response to the input pulse $x(m)$ as $r(m, n)$. The amplitude of $r(m, n)$ is proportional to $x(m)$ in magnitude and the wave form is similar to the impulse response. $r(m, n)$ is given by the following equation which appears at the output terminal from the time $n=m$.

$$
r(m, n)=x(m) h(n-m)
$$

where, $h(n-m)=0$ when $n-m<0$ as the causality should be satisfied.

Figure 1 shows the relation between the impulse response, the input sequence, the response to each input pulse and the output sequence as the sum of the responses to input pulses. This figure sums up the responses to the input pulses at each discrete time slot to make the response to input sequence. According to Fig. 1, the response to the input sequence $x(m),(m=0,1, \cdots, N-1)$ can be written 


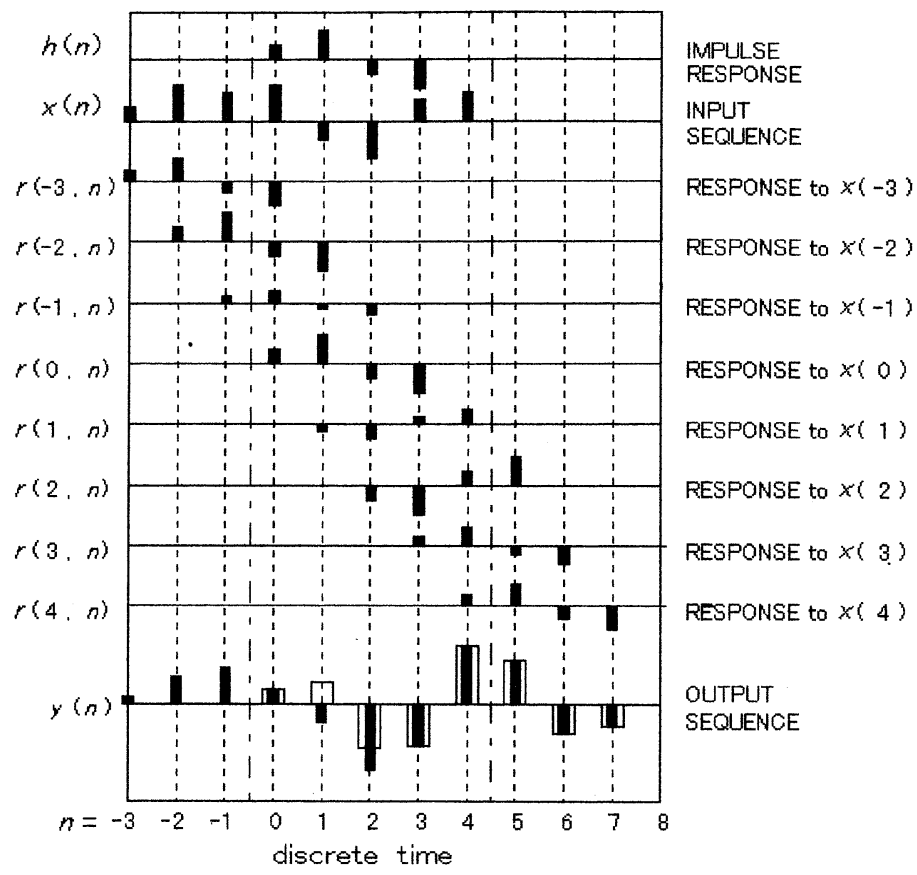

Fig. 1 Schematic explanation of the relation between the impulse response, input sequence and the output sequence as the sum of responses to each input pulse. White columns in the bottom shows the response for no input signal at $n<0$.

as the sum of $r(m, n)$ :

$$
y(n)=\sum_{m=0}^{N-1} r(m, n)=\sum_{m=0}^{N-1} x(m) h(n-m)
$$

This is the well known expression of the convolution of $x(n)$ and $h(n)$, but the responses to the input pulses before $n=0$ are neglected. The responses to $x(n),(-P<n<0)$ should be accounted for the composition of $y(n)$, if $x(n) \neq 0$ at $n<0$ and the length of impulse response is $P$. Therefore, the lower limit of the summation should be changed to $-(P-1)$ as shown in the following equation;

$$
\left.\begin{array}{l}
y(n)=\sum_{m=-(P-1)}^{N-1} x(m) h(n-m), \\
n=0,1, \cdots, N-1
\end{array}\right\}
$$

Here, it should be noted that the tail of $h(n-m)$ overflows the region $n=0,1, \cdots, N-1$ when $m+$ $P>N-1$. The overflowed part is discarded in the computation of the spectrum of $y(n)$ by $N$-points DFT. Accordingly, the impulse response $h_{m}(n)$ to an impulse input at $n=m$ changes by the value of $m$ as follows :

$$
h_{m}(n)= \begin{cases}h(n) & , n<N-m \\ 0 & , n>N-1-m\end{cases}
$$

Using this impulse response $h_{m}(n)$, the spectrum of an input pulse $x(m)$ is computed as follows:

$$
\begin{aligned}
\operatorname{DFT}\{r(m, n)\} \\
\quad=\sum_{n=0}^{N-1} x(m) h_{m}(n-m) \exp (-j 2 \pi k n / N) \\
=x(m) H_{m}(k) \exp (-j 2 \pi k m / N)
\end{aligned}
$$

where, $H_{m}(k)=\operatorname{DFT}\left\{h_{m}(n)\right\}$

The spectrum of the output sequence is the sum of Eq. (11) for every $m$.

$$
Y(k)=\sum_{m=-(P-1)}^{N-1} H_{m}(k) x(m) \exp (-j 2 \pi k m / N)
$$

This expression is utterly different from Eq. (3). The transfer function, that is the DFT of $h(n)$ can not be computed as the same manner as described using Eq. (3) through Eq. (6).

When the output sequence is multiplied by the time window function, the response to an input pulse can not be similar to the impulse response as the amplitude is modulated by the window function, moreover, the tail of response is cut off at the end of time window. The deformation of the output sequence by the multiplication of time window will be made clear in the following sections. 


\section{THEORETICAL COMPUTATION}

Here, we consider the effect of time window on the cross spectrum of the input and the output sequences. Figure 2 shows the composition of the system and Fig. 3 depicts schematically the relation between the input and the output sequences, the impulse response and the response to an input pulse. We have true input and output sequences $x(n)$ and $y(n)$ at the terminals 1 and 2 in Fig. 2, and the windowed input and output sequences $u(n)$ and $v(n)$ at the terminals 3 and 4 . The cross spectral technique is applied to the windowed sequences $u(n)$ and $v(n)$.

The pulse train expressed by the thin lines in the top of Fig. 3 stands for the impulse response of which the length is $N$ and the amplitude is assumed to be constant. The 2 nd sequence from the top stands for the input sequence where the pulse train multiplied by the time window is expressed using

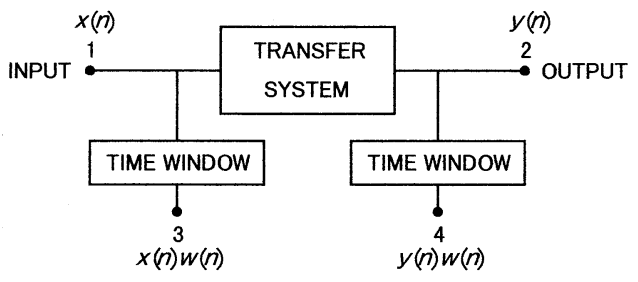

Fig. 2 Block diagram of a transfer system showing the input and the output terminal and the windowed signals.

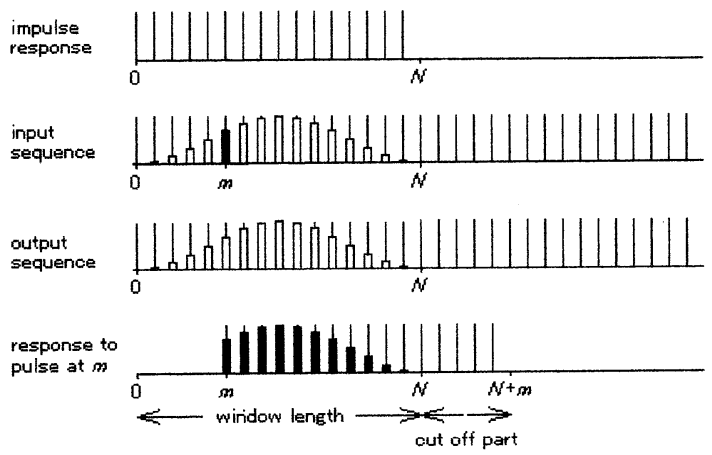

Fig. 3 Schematic representation of the relation between the impulse response $h(n)$, the input sequence $x(n)$, the output sequence $y(n)$ and the response to the pulse $x(m)$ in the input sequence. The white columns show the modulation in amplitude by time window. the white columns and a sample at a discrete time $m$ is expressed by a filled column. The 3 rd sequence from the top stands for the output sequence, where the windowed part is also shown by the white columns. The sequence in the bottom stands for the response to the input pulse at the discrete time $m$, where the windowed part is expressed by the thick lines.

In the first time, we take up an input pulse $x(m)$ at the discrete time $m$. The windowed $x(m)$ at the terminal 3 in Fig. 2 is written as $u(m)=x(m) w(m)$ using the window function $w(n)$. The response to input pulse $x(m)$ is the same as Eq. (9), but the wave form at the terminal 4 in Fig. 2 is different from Eq. (9) as the time window is multiplied. That is, the response to an input pulse $x(m)$ observed at the terminal 4 in Fig. 2 appears from the discrete time $m$ and is multiplied by the window function and is expressed as $v(n)=w(n) h(n-m) x(m)$. Therefore, the impulse response of the system to the pulse input at the discrete time $m$ observed at the terminal 4 is expressed as follows.

$$
g_{m}(n)=w(m+n) h(n)
$$

This impulse response is valid only for the pulse input at the discrete time $m$ and is cut at the time $n=N-m$ as $w(m+n)=0$ when $m+n \geq N$.

Therefore, the input and the output sequence observed at the terminals 3 and 4 are written as the followings.

At the terminal 3 :

$$
u(n)=w(m) x(m) \delta(n-m)
$$

At the terminal 4 :

$$
v(n)= \begin{cases}x(m) g_{m}(n-m) & n \geq m \\ 0 & n<m\end{cases}
$$

The spectrum of those sequences obtained by the $N$ points DFT is written as the followings.

$$
\begin{aligned}
& U(k)=w(m) x(m) \exp (-j 2 \pi k m / N) \\
& V(k)=x(m) G_{m}(k) \exp (-j 2 \pi k m / N)
\end{aligned}
$$

where,

$$
G_{m}(k) \text { is the } N \text { points DFT of } g_{m}(n) .
$$

Using those functions, the power spectrum of the input sequence $S_{U U}(k)$ and the cross spectrum of the input and the output sequences $S_{U V}(k)$ observed at the terminals 3 and 4 are written as the followings. 


$$
\begin{gathered}
S_{U U}(k)=w(m)^{2} \overline{x(m)^{2}} \\
S_{U V}(k)=w(m) \overline{x(m)^{2}} G_{m}(k)
\end{gathered}
$$

where, $x(m)$ is the $m$-th sample of the input sequence and

$$
\overline{x(m)^{2}} \text { is the many time average of } x(m)^{2}
$$

Here, we make the average of Eq. (18) for all the value of $m$. Then, the averaged value of $\overline{x(m)^{2}}$ becomes a constant $\overline{x^{2}}$ unless the input signal is a periodic signal synchronized to the time window. Therefore, the averaged values of Eqs. (18) and (19) can be written as the followings.

$$
\begin{gathered}
W_{U U}=\overline{S_{U U}(k)}=\frac{\overline{x^{2}}}{N} \sum_{m=0}^{N-1} w(m)^{2}=\frac{\overline{x^{2}}}{N} W_{X} \\
W_{U V}=\overline{S_{U V}(k)}=\frac{\overline{x^{2}}}{N} \sum_{m=0}^{N-1} w(m) G_{m}(k)
\end{gathered}
$$

The ratio of the cross spectrum to the power spectrum is the transfer function estimate in the cross spectral technique. The transfer function estimate $\hat{H}(k)$ is obtained by those equations :

$$
\hat{H}(k)=\frac{W_{U V}(k)}{W_{U U}(k)}=\frac{1}{W_{X}} \sum_{m=0}^{N-1} w(m) G_{m}(k)
$$

The IDFT of Eq. (22) is the impulse response estimate obtained by the cross spectral technique. The IDFT of each term of the above equation is easily carried out as $G_{m}(k)$ is the DFT of $g_{m}(n)$. Using the relation in Eq. (13), we can obtain the impulse response estimate $\hat{h}(n)$ as the following equation.

$$
\begin{aligned}
\hat{h}(n) & =\frac{1}{W_{X}} \sum_{m=0}^{N-1} w(m) w(m+n) h(n) \\
& =\frac{\sum_{m=0}^{N-1} w(m) w(m+n)}{\sum_{m=0}^{N-1} w(m)^{2}} h(n)
\end{aligned}
$$

The upper limit of the summation in the numerator is $N-1$ in this equation, but the substantial summation is carried out only from $m=0$ to $m=$ $N-1-n$ because $w(m+n)=0$ when $m+n \geq N$ and the upper limit can be changed to $N-1-n$. According to this result, the impulse response estimated by the cross spectral technique has the form of the product of the true impulse response and the auto correlation coefficient of the time window used, but the coefficient of the true impulse response is not true auto correlation coefficient as mentioned above. So, we use the following expression as the deforma- tion coefficient $D(n)$ hereafter, where the upper limit of the summation is written as $N-1-n$ to avoid possible confusion.

$$
D(n)=\frac{\sum_{m=0}^{N-1-n} w(m) w(m+n)}{\sum_{m=0}^{N-1} w(m)^{2}}
$$

\section{DEFORMATION WITH SIX TYPES OF TIME WINDOWS}

The deformation coefficients of six types of time window are computed. Those will be given in the following equations with the window functions:

\subsection{Rectangular Window}

Window function :

$$
w_{\mathrm{R}}(n)=1 \quad n=0 \text { to } N-1
$$

Deformation coefficient :

$$
D(n)=\left(1-\frac{n}{N}\right)
$$

4.2 Hanning Window

Window function :

$$
w_{\mathrm{N}}(n)=0.5\left(1-\cos \frac{2 \pi n}{N}\right)
$$

Deformation coefficient :

$$
D(n)=\frac{G(n)+H(n) / 2 \pi}{A^{2}+\frac{B^{2}}{2}+\frac{C^{2}}{2}}
$$

Where,

$$
\begin{gathered}
G(n)=\left(A^{2}+\frac{B^{2}}{2} \cos \frac{2 \pi n}{N}+\frac{C^{2}}{2} \cos \frac{4 \pi n}{N}\right)\left(1-\frac{n}{N}\right) \\
H(n)=\left(2 A B-\frac{B^{2}}{2}-\frac{2 B C}{3}\right) \sin \frac{2 \pi n}{N} \\
-C\left(A-\frac{4 B}{3}+\frac{C}{4}\right) \sin \frac{4 \pi n}{N} \\
A=0.5 \\
B=0.5 \\
C=0
\end{gathered}
$$

\subsection{Hamming Window}

Window function:

$$
w_{\mathrm{M}}(n)=\left(0.54-0.46 \cos \frac{2 \pi n}{N}\right)
$$

Deformation coefficient :

The same as Eq. (28)

Where, 


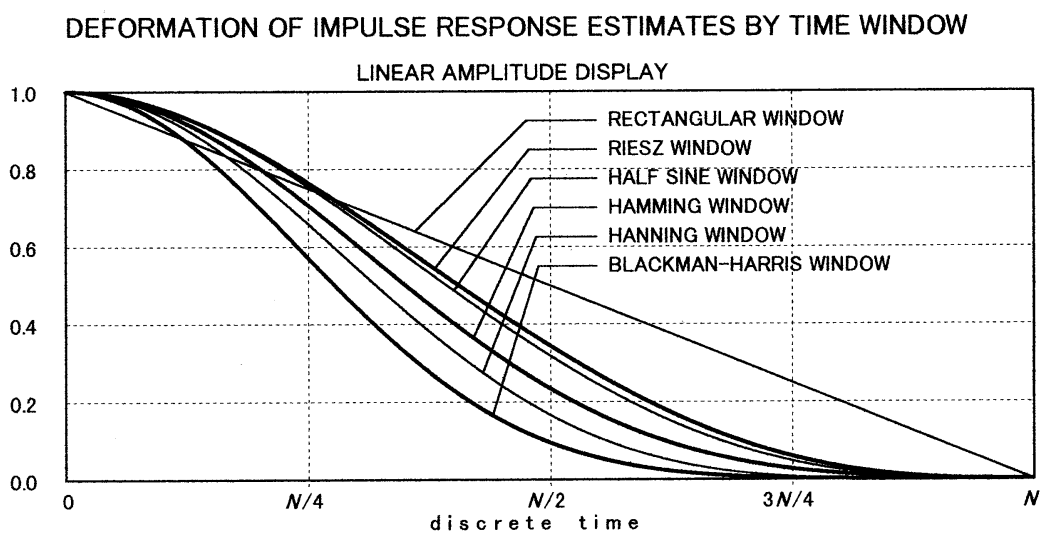

(a)

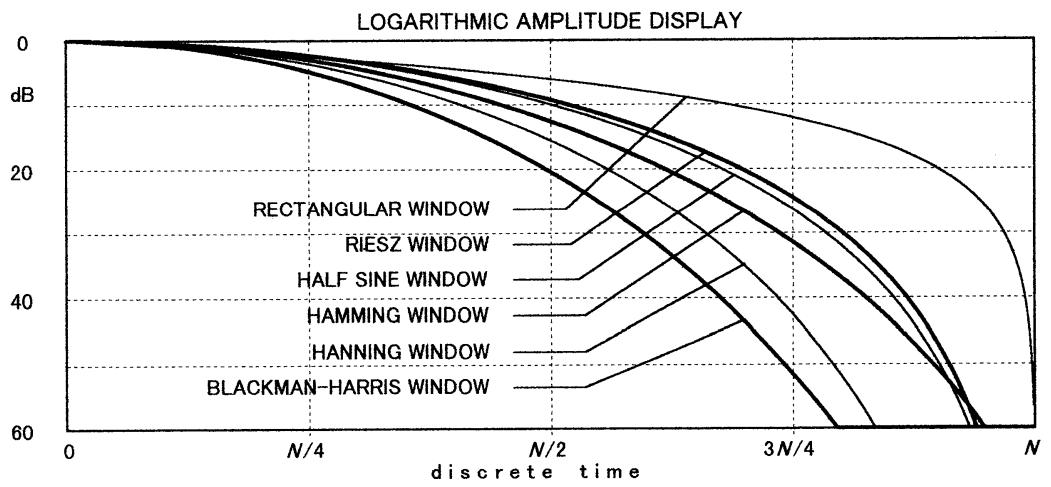

(b)

Fig. 4 Deformation coefficients theoretically computed for 6 types of time windows in linear scale (a) and in logarithmic scale (b).

$$
\begin{aligned}
& A=0.54 \\
& B=0.46 \\
& C=0
\end{aligned}
$$

\subsection{Blackman-Harris Window}

Window function :

$$
\begin{aligned}
& w_{\mathrm{B}}(n) \\
& =\left(0.423-0.498 \cos \frac{2 \pi n}{N}+0.0792 \cos \frac{4 \pi n}{N}\right)
\end{aligned}
$$

Deformation coefficient: The same as Eq. (28) Where,

$$
\begin{aligned}
& A=0.423 \\
& B=0.498 \\
& C=0.0792
\end{aligned}
$$

\subsection{Half Sine Window}

Window function :

$$
w_{\mathrm{S}}(n)=\sin \left(\frac{\pi n}{N}\right) w_{\mathrm{R}}(n)
$$

Deformation coefficient :

$$
D(n)=\cos \frac{\pi n}{N}\left(1-\frac{n}{N}\right)+\frac{1}{\pi} \sin \frac{\pi n}{N}
$$

\subsection{Riesz Window}

Window function:

$$
w_{Z}(n)=1-\left(1-\frac{2 n}{N}\right)^{2}
$$

Deformation coefficient :

$$
D(n)=5\left(2-\frac{3}{N}+2 \frac{n^{2}}{N^{2}}\right)\left(1-\frac{n}{N}\right)^{3}-9\left(1-\frac{n}{N}\right)^{5}
$$

Figures 4 (a) and (b) show the deformation coefficients computed by the above equations with the linear scale and the $\mathrm{dB}$ scale respectively. 


\section{VERIFICATION BY COMPUTER SIMULATION}

The results described in the preceding section are verified by computer simulation for six types of time windows using a part of uniformly distributed random sequence of which the mean value is zero as the source signal $x(n)$. In the simulation, the output sequence $y(n)$ of the transfer system is first computed as the convolution of $x(n)$ and the impulse response $h(n)$. Next the input and the output sequences of length $N=512$ are multiplied by the window function $w(n)$ of the same length and 512 points DFT's of $x(n) w(n)$ and $y(n) w(n)$ are computed and the cross spectrum is divided by the power spectrum of input sequence to obtain the transfer function estimate. To be exact, $M$ times average of $[\operatorname{DFT}\{x(n) w(n)\}] *[\operatorname{DFT}\{y(n) w(n)\}]$ is used as the cross spectrum and the same times average of $|\operatorname{DFT}\{x(n) w(n)]|^{2}$ is used as the power spectrum. The cross spectrum is divided by the power spectrum to make the transfer function estimate. Finally impulse response estimate is made by the IDFT of the transfer function estimate. The averaging time $M$ will be shown in each specific case.

In the first investigation, two kinds of impulse responses are used. One of them is a sinusoidal signal and another one is a part of random sequence of which the mean value is zero. Both the impulse responses do not decay with time and the length is

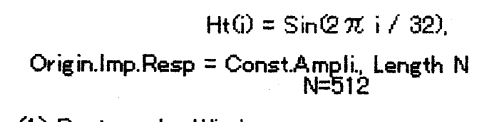

10000 iterations for Averaging
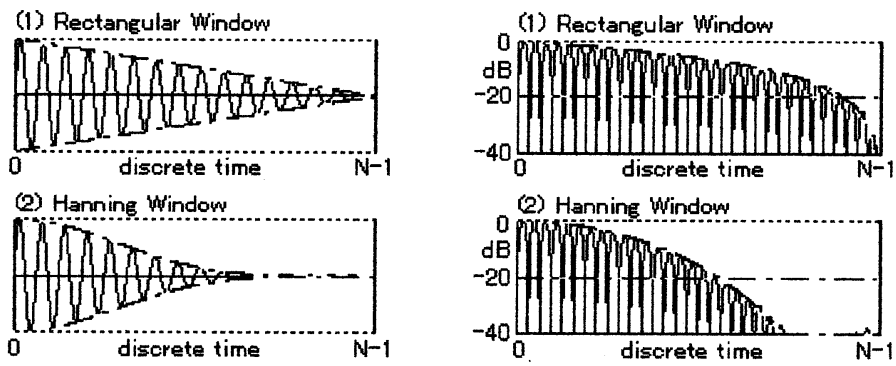

(2) Hanning Window
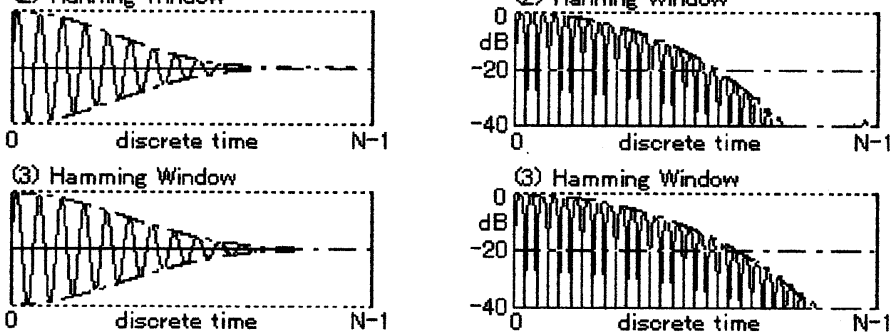

(3) Hamming Window

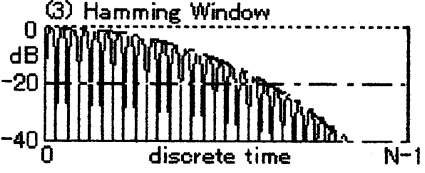

(4) Blackman-Harris Window

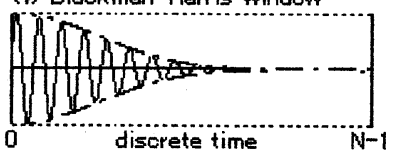

(4) Blackman-Harris Window

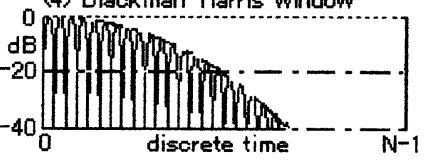

(5) Half Sine window

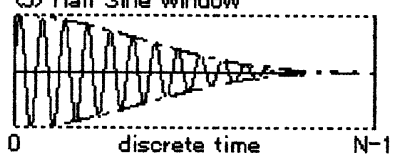

(5) Half Sine window

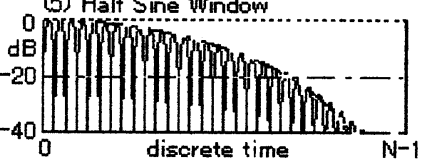

(6) Riese Window

(6) Riese window

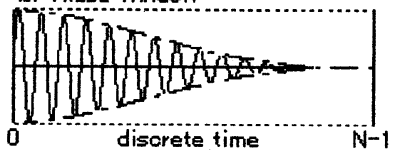

(a)

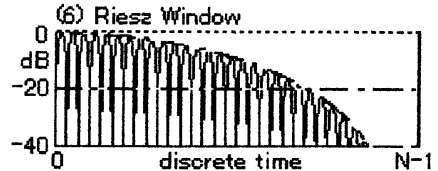

(b)

Fig. 5 The estimated impulse responses and the theoretically computed envelopes of impulse response estimates for six types of time windows. The original impulse response is constant amplitude sinusoidal wave of length $N(=512)$. (a) The wave form, (b) The squared value in $\mathrm{dB}$ scale. 
512 which is the same as the length of time window. The averaging time $M$ is 10,000 in the first investigation. Many time average is necessary to suppress the noise to the level lower than $-40 \mathrm{~dB}$ as the response to the signal input before the time window has the same level as that to the signal input during the period of time window. The former response acts as the noise and the latter response only is effective in the impulse response estimation.

Figures 5 and 6 show the impulse responses obtained by the first investigation for six types of time windows : the Rectangular window, the Hanning window, the Hamming window, the Blackman-Harris window, the half sine window and the Riesz window. In those figures, shown in the left hand side are wave forms of the impulse responses in the linear scale and those in the right hand side is the squared wave forms in the $\mathrm{dB}$ scale. The details in the large amplitude region can be observed in the linear scale figures of the left hand side and the details in the small amplitude region in the $\mathrm{dB}$ scale figures. If an exact impulse response could be estimated, the amplitude of the estimated impulse response should be constant as the amplitude of the original impulse response used to make the output sequence was constant. But the estimated ones decay in the time as the same manner as theoretically expected given by $D(n)$ 's in Eqs. (26) through (34). The envelopes computed by those equations are drawn by the decaying chain curves
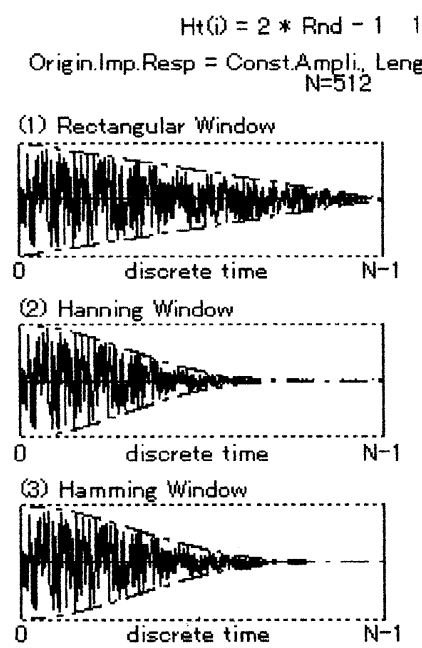

(4) Blackman-Harris Window

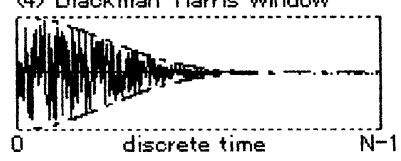

(5) Half Sine window
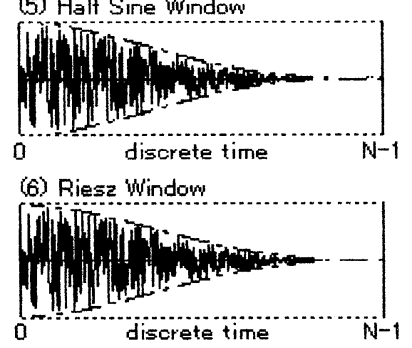

(a)
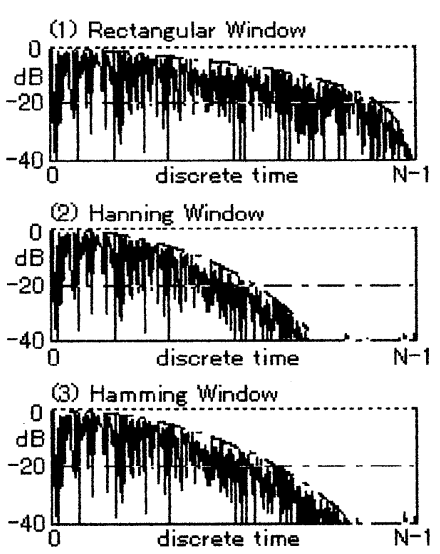

(4) Blackman-Harris Window

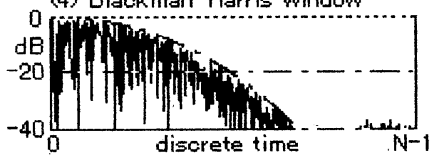

(5) Half Sine Window

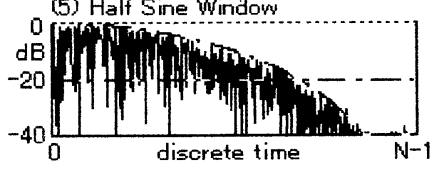

(6) Riesz Window

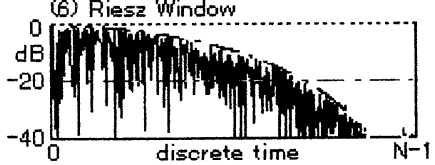

(b)

Fig. 6 The estimated impulse responses and the theoretically computed envelopes of impulse response estimates for six types of time windows. The original impulse response is uniformly distributed random noise of length $N(=512)$. (a) the wave form, (b) the squared value in $\mathrm{dB}$ scale. 


\section{K. KIDO et al. : DEFORMATION OF IMPULSE RESPONSE BY TIME WINDOW}

and the impulse response estimates are given by wave forms. Those figures indicate that the change in the envelope of impulse response estimated by the cross spectral technique coincides with the theoretically expected change for every kind of time window.

It is also observed from those figures that the deformation of impulse response in the process of estimation has no relation with the waveform of the impulse response.

Figure 7 shows the other examples of estimated impulse response using the six types of time windows of length $N(=512)$, where the impulse response is an exponentially decaying sinusoidal wave. We define here the length of the impulse response as the time in which the amplitude of the impulse response attenuates to $1 / 1,000$ of the initial value, that is the reverberation time in the room acoustics. The length of the impulse response is 2 times of the window length $N$ in the examples in Fig. 7. In the other word, the time constant of the impulse response is $2 / 6.9$ of the window length, that is $2 \times N / 6.9=148$. The averaging time $M$ is 500 in this case. The noise due to the response to the input signal before the windowed period is less in this example than in the former examples of Figs. 5 and 6 as the impulse response decays with time.

In each graph (1) through (6), the outer dotted lines indicate the envelope of original impulse response, the inner broken lines the envelope of the estimated impulse response computed by one of the theoretically obtained equations through (26) to
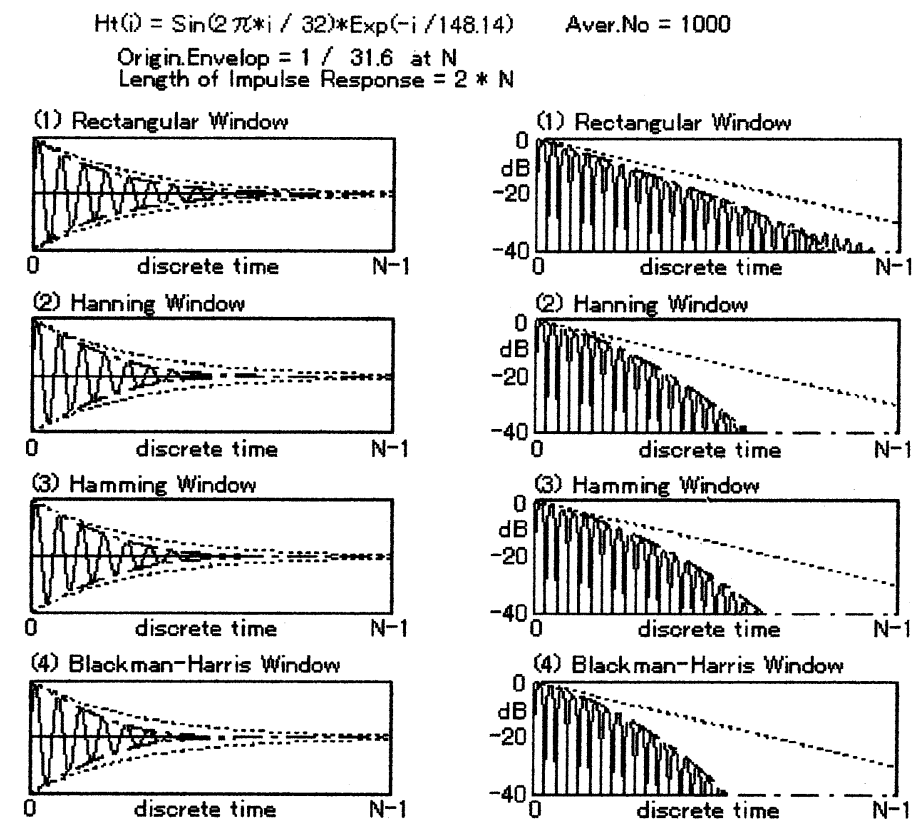

(2) Hanning Window
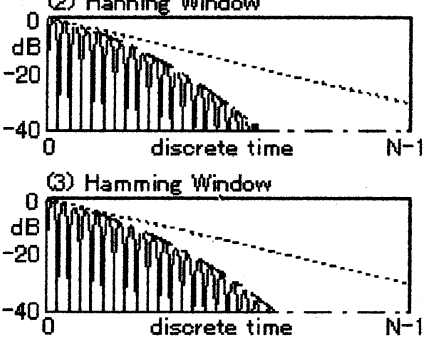

(4) Blackman-Harris Window
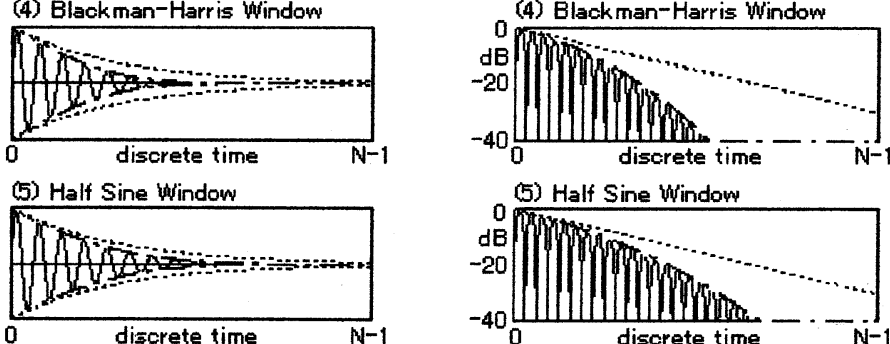

(5) Half Sine Window

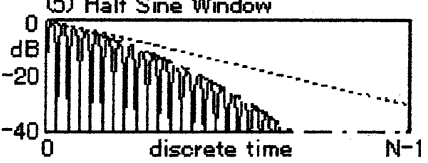

(6) Riese Window

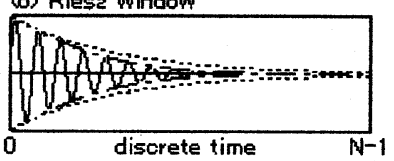

(a)

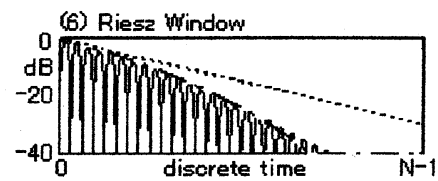

(b)

Fig. 7 The estimated impulse responses and the theoretically computed envelopes for six types of time windows. The outside dotted lines are the envelope of the original impulse response which attenuates $60 \mathrm{~dB}$ in 2 times of the window length. (a) the wave form, (b) the squared value in $\mathrm{dB}$ scale. 
(34) and the wave form is the impulse response estimate obtained by the cross spectral technique. The inner broken lines overlap with the envelope of the estimated impulse response and the difference can hardly be seen.

The length of the impulse response is 2 times of the window length in the examples shown in Fig. 7. But in the usual applications, the length of the impulse response is not so much longer than the window length. And we take up the examples shown in Fig. 8 where the length of the impulse response is a half window length. The averaging time is 100 in this example as the response to the signal input before the time window is less than former examples. The deformation of the impulse response seems to be negligible in the linear scale expression as the impulse response is deformed in the region of the small amplitude. But, in the $\mathrm{dB}$ expression, we can see the differences between the true decay curves and the estimated decay curves. The reverberation time or the time constant of the estimated impulse response is shorter than the original ones in all the time windows. Among them, the rectangular window gives the best result and the Blackman-Harris window gives the worst result.

\section{SUMMARY AND DISCUSSIONS}

This paper first points out two problems in the superficial explanation of cross spectral technique. The first one is the possibility of confusion of the linear convolution and the circular convolution. The second one is the overlooking of the window
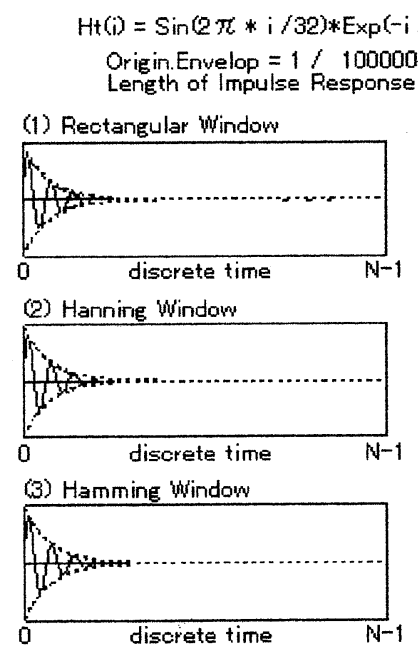

(4) Blackman-Harris Window

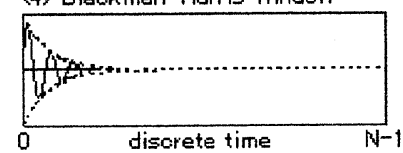

(5) Half Sine window
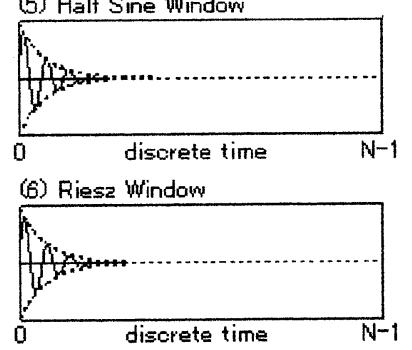

(a)
AverNo. $=100$

\section{$N N=256$}
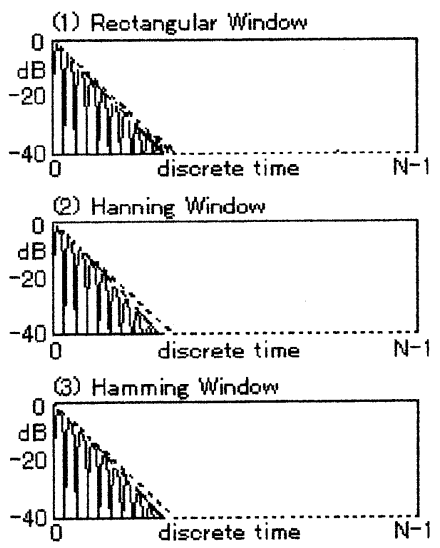

(4) Blackman-Harris Window

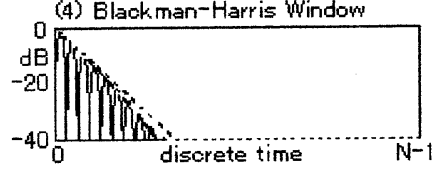

(5) Half Sine Window
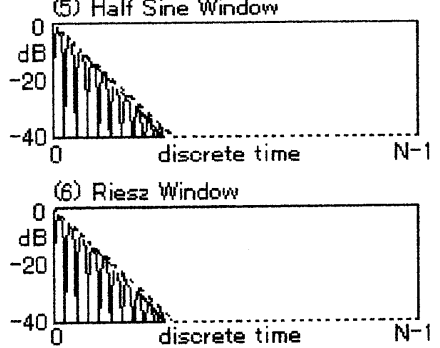

(b)

Fig. 8 The estimated impulse responses and the theoretically computed envelopes for six types of time windows. The outside dotted lines are the envelope of the original impulse response which attenuates $60 \mathrm{~dB}$ in a half window length. (a) the wave form, (b) the squared value in $\mathrm{dB}$ scale. 
function multiplied in the time domain.

Before the theoretical formulation of deformation coefficients, some preliminary discussions are described to make clear the succeeding theoretical derivation, where the schematic explanation of the convolution and the DFT are given. Then, how the impulse response is deformed is theoretically formulated in a general form.

Based on the general expression, the deformation coefficients for six types of time windows are given and illustrated in Fig. 4. The results of the theoretical formulation are verified by the computer simulation for each type of time window using an impulse response which does not decay in time. And the change in the envelope of impulse response is illustrated for two cases using exponentially decaying impulse responses, where the length of impulse response is 2 times and a half times of the window length respectively. A significant shortening is observed in the estimated reverberation time or time constant when the length of impulse response (reverberation time) is longer than $1 / 4$ of the length of time window.

According to the results, the deformation is bigger with the windows of lower side lobe level such as the Blackman-Harris window than with the windows of higher side lobe level such as the rectangular window or the Riesz window. The superiority of the Riesz window in the impulse response estimation was shown by the group of one of the authors as the experimental study. ${ }^{5-7}$ The results of this paper coincide with them. The deformation by the Riesz window is nearly the same as that by the half sine window as the shapes of those windows in the time domain are very similar.

Watching to the very initial part of the deformation coefficient shown in Fig. 4 (a), the deformation by the Riesz and half sine window is less than that by widely used time windows such as the Hanning or Hamming windows. We can expect that the Riesz and half sine windows produce good results if the length of impulse response is less than $1 / 4$ of window length. But the deformation coefficients of those time window decrease suddenly in the middle part of time window.

From the same figure, it is also expected that the rectangular window gives the best result among those windows if the length of impulse response is very long.

It is interesting that the evaluation of the time window in the view point of the deformation of impulse response in the time domain shows reverse tendency to the evaluation in the view point of the spectrum side lobes in the frequency domain. This tendency can be qualitatively explained by the difference in the effective length of time window.

The deformation of impulse response by the time window is negligible if the length of impulse response is shorter than about $1 / 4$ of the window length. The results given in this paper is considered to be effective only in the case of long impulse response. More detailed investigation will be necessary to made quantitatively clear the relation between the properties of time window and the estimation error.

This paper deals with a special case where the window length in the number of sampled data is the same as the number of points for the DFT as it is common in the cross spectral technique widely used to estimate the transfer function and this algorithm is installed with most of the FFT analyzer. As the result, we come up against the problem to carry out the deconvolution of a sequence made by linear convolution using the algorithm in which the convolution is inevitably the circular convolution.

There is a well known method to compute the exact convolution in the frequency domain excluding the effect of circular convolution, ${ }^{8-10)}$ where the sequence of null date are followed to the input and the output sequences as shown in Fig. 9 (a). But this method is applicable only to the computation of convolution. The result is not improved in the impulse response estimation. The impulse response estimated using such the type of time window is the same as that obtained using the full length time window. Another method is necessary to obtain exact impulse response.

There can be an idea in which the cross spectrum of the shortened input sequence followed by a sequence of null date and the full length output

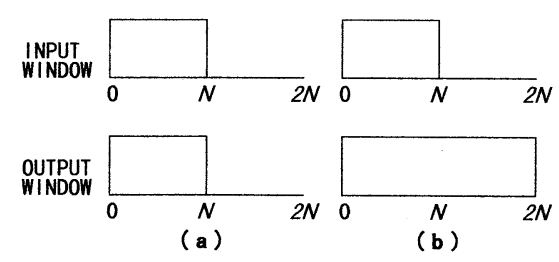

Fig. 9 Schematic representation of the shortened windows to avoid the effect of circular convolution in DFT. 
sequence is used as shown in Fig. 9 (b). ${ }^{10)}$ The length of null sequence should be equal to or longer than the length of impulse response. The time window used in this method is limited to the rectangular window. Taking this type of input window, all the responses to the samples in the input window are contained in the output window and there is no problem of circular convolution nor cutting off the tail of response. An exact impulse response can be obtained by this method. But this method is not necessarily practicable, because quite many averaging time is necessary in this method as a lot of components incoherent to the signal in the input window is in the output sequence cut out by the output window. Those components behave as noise.

Anyhow, this paper aims to make clear the unrecognized problem on the use of time window in the conventional cross spectral technique widely used. How to estimate an exact impulse response is another problem which has been investigated and being developed even now.

There remain some problems as follows :

More investigation will be necessary to make numerically clear the relation between the impulse response estimation error and the length of time window for every type of time window. The compensation of the deformation in the estimated impulse response dividing by the deformation coefficient is considered to be possible, but the division by the deformation coefficient produces a big increase in amplitude near the end of the time window where is usually some noise. The compensation does not necessarily improve the estimation. More investigation is necessary for the effective compensation.

The investigation in this paper is limited to the deformation of the impulse response, but the deformation should be in the transfer function. It may be necessary to discuss the effect of time window in the frequency domain.

The time window is used not only in the cross spectral technique but also in the spectrum estimation by the LPC analysis for the computation of covariance or correlation. It may be explained by the same way as described in this paper that the stable estimation is achieved by the correlation method. The relation between the estimated band width of poles and the window function will be an interesting problem.

\section{CONCLUSIONS}

This paper describes the effect of time window on the impulse response estimate in the process of estimation by the cross spectral technique. Why and how the impulse response is deformed is first theoretically explained and the results are verified by the computer simulation. There are two causes. One of them is that the tail of the response to the input signal captured in the input time window flows out from the end of the output time window. Another one is that the amplitude of the input and the output signals are modulated by the window function.

How the estimated impulse response is deformed is theoretically formulated for general expression of window function. The specific deformation coefficients for six types of time windows are given in the formulae and in the illustrations. The theoretical results are verified by the computer simulation. According to the results obtained, the envelope of impulse response changes considerably in the process of estimation by the cross spectral technique unless the window length is much longer than the length of impulse response.

\section{REFERENCES}

1) N. Aoshima, "Computer-generated pulse signal applied for sound measurement," J. Acoust. Soc. Am. 69, 1484-1488 (1981).

2) Y. Suzuki, F. Asano, H. Kim, and T. Sone, "An optimum computer-generated pulse signal suitable for the measurement of very long impulse response," J. Acoust. Soc. Am. 97, 1119-1123 (1995).

3) K. Kido, T. Yamazaki, H. Itakura, and J. Liu, "A method for estimation of impulse response only by averaging," J. Acoust. Soc. Jpn. (J) 49, 153-159 (1993) (in Japanese).

4) F. J. Harris, "On the use of windows for harmonic analysis with the discrete Fourier transform," Proc. IEEE 66, 51-83 (1978).

5) Y. Nagata, J. Liu, M. Abe, and K. Kido, "Effect of a time window on the coherence function and the impulse response estimated by the cross spectral technique,” J. Acoust. Soc. Jpn. (J) 45, 950-956 (1989) (in Japanese).

6) C. Kim, M. Abe, and K. Kido, "Comparative study of methods for the estimation of impulse response," Proc. 11. ICA Paris 1983, 297-300 (1983).

7) M. Abe, C. Kim, and K. Kido, "Effect of time window on accuracy of estimation of impulse response using white noise," Proc. 11. ICA Paris 1983, 305-308 (1983).

8) L. R. Rabiner and B. Gold, Theory and Application of Digital Signal Processing (Prentice-Hall Inc., 


\section{K. KIDO et al. : DEFORMATION OF IMPULSE RESPONSE BY TIME WINDOW}

Englewood Cliffs, New Jersey, 1975), p.61.

9) A. V. Oppenheim and R. W. Schafer, Digital Signal Processing (Prentice-Hall Inc., Englewood Cliffs, New Jersey, 1975), p.110.

10) M. Fukushima, K. Ohe, K. Kido, H. Suzuki, and T. Ono, "The effect of short time windows for impulse response estimation with cross spectral technique," Tech. Rep. IEICE, EA93-34 (1993) (in Japanese).

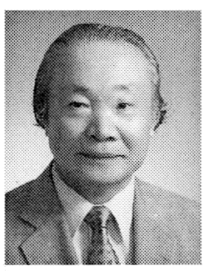

Ken'iti Kido received the BS degree in electrical engineering in 1948 and the Dr. Eng. Degree in 1962, both from Tohoku University, Sendai, Japan. From 1963 to 1976, he was a Professor of Acoustics in the Research Institute of Electrical Commumcation, Tohoku University. From 1976 to 1990 , he was a Director of the Research Center for Applied Information Science, Tohoku University. After retiring in 1990, he received the degree of Professor Emeritus from the same university. Now, he is a part time professor at the Department of Computer Science Chiba Institute of Technology and Chairman of Ontek R\&D Co. Ltd., Yokohama Japan. $\mathrm{He}$ has been engaged in the research on electroacoustics, active noise control, speech recognition and the signal processing in acoustics. Dr. Kido is a fellow of the Acoustical Society of America, a corresponding member of the Institute of Noise Control Engineering of USA, an honorary member of the Acoustical Society of Japan and the Founder Chairman of the Western Pacific Commission for Acoustics. He is also a member of the Information Processing Society of Japan, the Acoustical Society of Korea and some other academic societies.

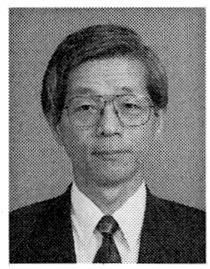

Hideo Suzuki received MS in 1967 from Tohoku University and Ph.D. in 1981 from Pennsylvania State University. He worked for Mitsubishi Electric in the field of loudspeaker development during 1967-1979. From 1981 to 1985, he worked for CBS Inc. in the field of piano acoustics (for Steinway and
Sons). From 1985, he worked for Ono Sokki developing sound and vibration measurement analysis systems. From 1992, he has been the president of Ontek R\&D, a consulting firm on sound and vibration. Dr. Suzuki is a fellow of the Acoustical Society of America, a member of the Acoustical Society of Japan, Institute of Noise Control Engineering of Japan, Japan Society of Mechanical Engineers.

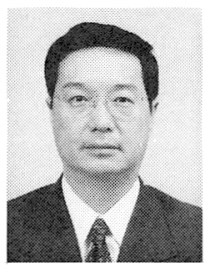

Takahiko Ono received the BA degree from Keio University, Faculty of Business and Commerce, Tokyo, Japan in 1974. In 1989, he received the degree of Dr. Eng. in Electrical Engineering from Tohoku University, Sendai, Japan. In 1976, he joined Ono Sokki Co. Ltd., as the product planning engineer. He was named as a member of the board in 1983. Since 1991, he has been President and Chief Executive Officer of Ono Sokki Co. Ltd. Besides his business, he is now a part-time teacher at Tokyo University of Agriculture and Technology. $\mathrm{He}$ is a member of the Acoustical Society of Japan, the Japan Society of Mechanical Engineers, the Institute of Noise Control Engineering of Japan, the Institute of Electrical Engineers of Japan and the Institute of Electronics, Information and Communication Engineers of Japan.

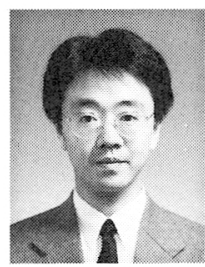

Manabu Fukushima received the $\mathrm{BE}$ degree in electronic engineering from Chiba Institute of Technology in 1989. He had been at Fuji Xerox Co., Ltd. as an engineer from 1989 to 1991. Since 1991, he is a research associate at Chiba Institute of Technology. $\mathrm{He}$ started working on the computer communication at the CIT and now he works on the research on digital signal processing. $\mathrm{He}$ is a member of the Acoustical Society of Japan, the Institute of Electronics, Information and Communication Engineers of Japan and the Information Processing Society of Japan. 